\title{
Physiology and immunology of the cholinergic antiinflammatory pathway
}

\author{
Kevin J. Tracey \\ The Feinstein Institute for Medical Research, Manhasset, New York, USA.
}

\begin{abstract}
Cytokine production by the immune system contributes importantly to both health and disease. The nervous system, via an inflammatory reflex of the vagus nerve, can inhibit cytokine release and thereby prevent tissue injury and death. The efferent neural signaling pathway is termed the cholinergic antiinflammatory pathway. Cholinergic agonists inhibit cytokine synthesis and protect against cytokine-mediated diseases. Stimulation of the vagus nerve prevents the damaging effects of cytokine release in experimental sepsis, endotoxemia, ischemia/reperfusion injury, hemorrhagic shock, arthritis, and other inflammatory syndromes. Herein is a review of this physiological, functional anatomical mechanism for neurological regulation of cytokine-dependent disease that begins to define an immunological homunculus.
\end{abstract}

The germ is nothing; the terrain is everything.

- Louis Pasteur (on his deathbed)

\section{Historical perspective of the cytokine theory of disease}

For more than 1,500 years, the predominant medical doctrine of disease causation was the humoral theory of disease. As posited by Galen (Claudius Galenus of Pergamum, 129-200 AD), this theory held that disease was caused by an imbalance in bodily humors. Produced by internal organs, the humors mediated healthful effects when their levels were properly regulated and balanced. This theory was not displaced until advances in microscopy and microbiology enabled Rudolf Virchow to formulate the theory of cellular pathology and Louis Pasteur the germ theory of disease. Research into the causes of illness turned outward as a growing body of investigation focused on exogenous rather than endogenous factors in disease causation. Pasteur's deathbed remark, however, belied the notion that germs could tell the whole story. It was a tacit acknowledgment to Claude Bernard, who advocated that health was due to equilibrium in the "milieu interieur" and maintained by "a continuous and delicate compensation, established as by the most sensitive of balances" (Lessons on the phenomena of life common to animals and vegetables).

Common wisdom in the 1970s dictated that the immune response to pathogens or injury was uniformly for the good. Every facet of the immune response was believed to have a role (albeit some unknown) in eradicating invaders and neutralizing the potential for damage. Certainly, or so the reasoning went, the body would not use its own cells to make potentially toxic molecules in quantities large enough to injure tissues or even kill. The development of shock, tissue injury, circulatory collapse, hemorrhagic necrosis, multiple organ failure, and other physiological calamities associated with bacterial infection were attributed to the direct action of pathogenic toxins, not the host response.

A few dared to challenge this view, notably Lewis Thomas, who wrote in his classic 1975 book The lives of a cell, "When we sense lipopolysaccharide, we are likely to turn on every defense at our

Nonstandard abbreviations used: $\mathrm{ACTH}_{1-24}$, adrenocorticotropic hormone; HMGB1, high-mobility group box 1 ; nAChR, nicotinic AChR.

Conflict of interest: The author is a consultant for Critical Therapeutics. Citation for this article: J. Clin. Invest. 117:289-296 (2007). doi:10.1172/JCI30555. disposal; we will bomb, defoliate, blockade, seal off, and destroy all the tissues in the area. All of this seems unnecessary, panicdriven ... The self-disintegration of the whole animal that follows a systemic injection can be interpreted as a well-intentioned but lethal error. The mechanism is itself quite a good one, when used with precision and restraint (1)." Scientific advances would soon prove his statement to be unerringly prescient. Early investigators exploring physiological mechanisms of fever, weight loss, and acute phase responses to acute and chronic infection led the way in changing beliefs about disease pathogenesis. They gave evidence that proteins produced by macrophages and other immune cells, not pathogens, caused tissue damage and disease syndromes in experimental animals. Studies in the 1980s of these proteins, newly named cytokines (including, for example, IL-1, IL-6, and $\mathrm{TNF}$ ), revealed that they possessed pleiotropic biological activities that were either beneficial or injurious.

From this emerged the cytokine theory of disease, the concept that cytokines produced by the immune system can cause the signs, symptoms, and damaging aftereffects of disease (Figure 1) (reviewed in ref. 2). Consider as one example the case of TNF, a cytokine implicated as a necessary and sufficient mediator of lethal septic shock caused by infection with Gram-negative bacteria. TNF is produced during Gram-negative bacteria-induced acute septic shock (3). Administration of TNF to healthy mammals (including humans) reproduces the hemodynamic, metabolic, immunological, and pathological manifestations of the disease (4), and gene knockout or pharmacological blockade of TNF activity prevents the development of lethal septic shock (5). Other pathophysiological activities ascribed to TNF were the capacity to cause fever and localized inflammation $(4,6)$. Clinical successes in the 1990s using drugs that specifically inhibit TNF for patients with inflammatory bowel disease or rheumatoid arthritis directly implicated a pathogenic role of this cytokine in other diseases (7-9) and validated in humans the fundamental premise of the cytokine theory of disease.

Today, drugs that specifically inhibit or neutralize endogenous cytokines are in widespread use. Anti-TNF- and anti-IL-1-based therapeutics have been administered to millions of patients, many of whom enjoy a significantly improved quality of life that was simply not achievable with other treatments. Dose-response and safety studies have defined the therapeutic indexes for these 


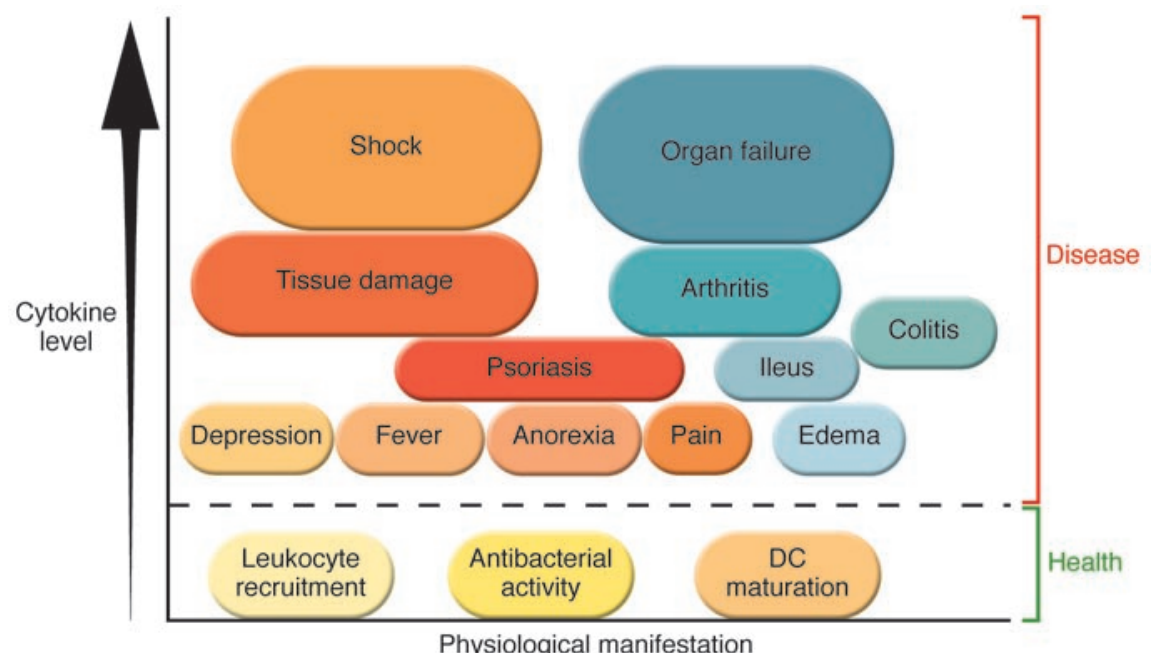

Figure 1

The cytokine theory of disease. Health requires that cytokine production is balanced: low levels are required to maintain homeostasis. Overproduction of some cytokines causes diseases that span the range of severity from mild to lethal. Some diseases can develop as a result of several different cytokines, as occurs, for example, in arthritis, which can be mediated by TNF, IL-1, or HMGB1. Other diseases may be specifically caused by individual cytokines, as occurs, for instance, in acute septic shock, with lethal hemorrhagic tissue injury mediated by an overproduction of TNF.

agents, which can be administered in doses that reduce cytokine activity to levels that are compatible with health without causing significant immunosuppression. Additional clinical trials are in progress, and it is likely that therapeutics based on the cytokine theory of disease will be approved for other indications. Incidentally, it also seems that ancient Greek concepts were not far off, since our modern theories about disease causation implicate an imbalanced production of bodily substances.

\section{Antiinflammatory humoral mechanisms restrain cytokine production}

The discovery that cytokines have the capacity to cause disease focused a new field of investigation on the physiological control mechanisms that maintain health by restraining or counterregulating cytokine release. Many endogenous antiinflammatory mediators can prevent cytokine-mediated disease (reviewed in ref. 10). During the earliest stages of microbial encroachment, these molecules begin to accumulate at the site of inflammation or in the blood. When sufficient concentrations are achieved, proinflammatory cytokine levels are inhibited, the magnitude of the inflammatory response is dampened, and damage is prevented. These antiinflammatory mediators function through humoral mechanisms by virtue of their action along concentration gradients in the extracellular milieu. It is a redundant system that constrains the potentially injurious cytokine response to operate within a safe range. The importance of these antiinflammatory pathways is evident in studies of animals deficient in specific mediators, which develop escalating disease. For example, IL-10-deficient mice have increased susceptibility to both inflammatory bowel disease and adjuvant arthritis, and rat strains that mount a diminished corticotropin-releasing factor (a polypeptide hormone and neurotransmitter) response are prone to developing adjuvant-induced inflammatory diseases (11-13).
Knowledge of humoral antiinflammatory mechanisms furthered the research and development of therapeutics, including glucocorticoids and other cytokine inhibitors that protect against cytokine-mediated tissue damage in humans.

\section{The cholinergic antiinflammatory pathway}

Recently, my colleagues and I described an alternative mechanism for cytokine control based on the structure of the nervous system (Figure 2) (14). We reasoned that, since the CNS coordinates major physiological responses via innervated circuits, it might also use neural input to control a potentially deadly cytokine response, especially one capable of inflicting enough damage to disrupt or shut down essential physiological systems. In classical physiological systems, the sensory projections of the autonomic nervous system provide input to brain networks about essential bodily functions. These elicit a coordinated neural output from the CNS to maintain homeostasis for parameters as varied as heart rate, blood pressure, digestion, body temperature, organ perfusion, and blood glucose levels. Accordingly, it seemed possible to posit the existence of a comparable mechanism to control cytokine release that could, at least in theory, function as an extremely fast, reflex-like antiinflammatory pathway controlled by brain networks (14).

That the vagus nerve might be a candidate conduit through which the brain could exert control over cytokine expression seemed plausible for several reasons. First, this cranial nerve, which meanders through the thorax and abdomen and has been termed the single most important nerve in the body, is a primitive extension of the CNS to the visceral organs, including organs of the reticuloendothelial system, such as the spleen and liver, that are major sources of damaging cytokines. Second, sensory fibers traveling with the vagus nerve transmit information to the brain that is both necessary and sufficient for initiating fever and other responses to injury or infection $(15,16)$. This could serve as the sensory arm of the inflammatory reflex, a neural loop in which afferent inflammatory signals activate an opposing motor response in the vagus nerve that suppresses cytokine production in order to limit or prevent damage (14). Third, an accidental discovery revealed that intracerebral administration of a molecule that inhibited TNF production also increased efferent vagus nerve activity and inhibited inflammation outside the CNS (17). These possibilities were studied directly by stimulating the vagus nerve in experimental animals with cytokine disease.

Stimulation of vagus nerve signals was shown to significantly inhibit TNF release in animals receiving lethal amounts of endotoxin (18). Subsequent work established that vagus nerve signaling inhibits cytokine activities and improves disease endpoints in experimental models of sepsis, ischemia/reperfusion, hemorrhagic shock, myocardial ischemia, ileus, experimental arthritis, and pancreatitis (17-25). The cellular molecular mechanism for inhibition of cytokine synthesis is attributable to acetylcholine (ACh), the 


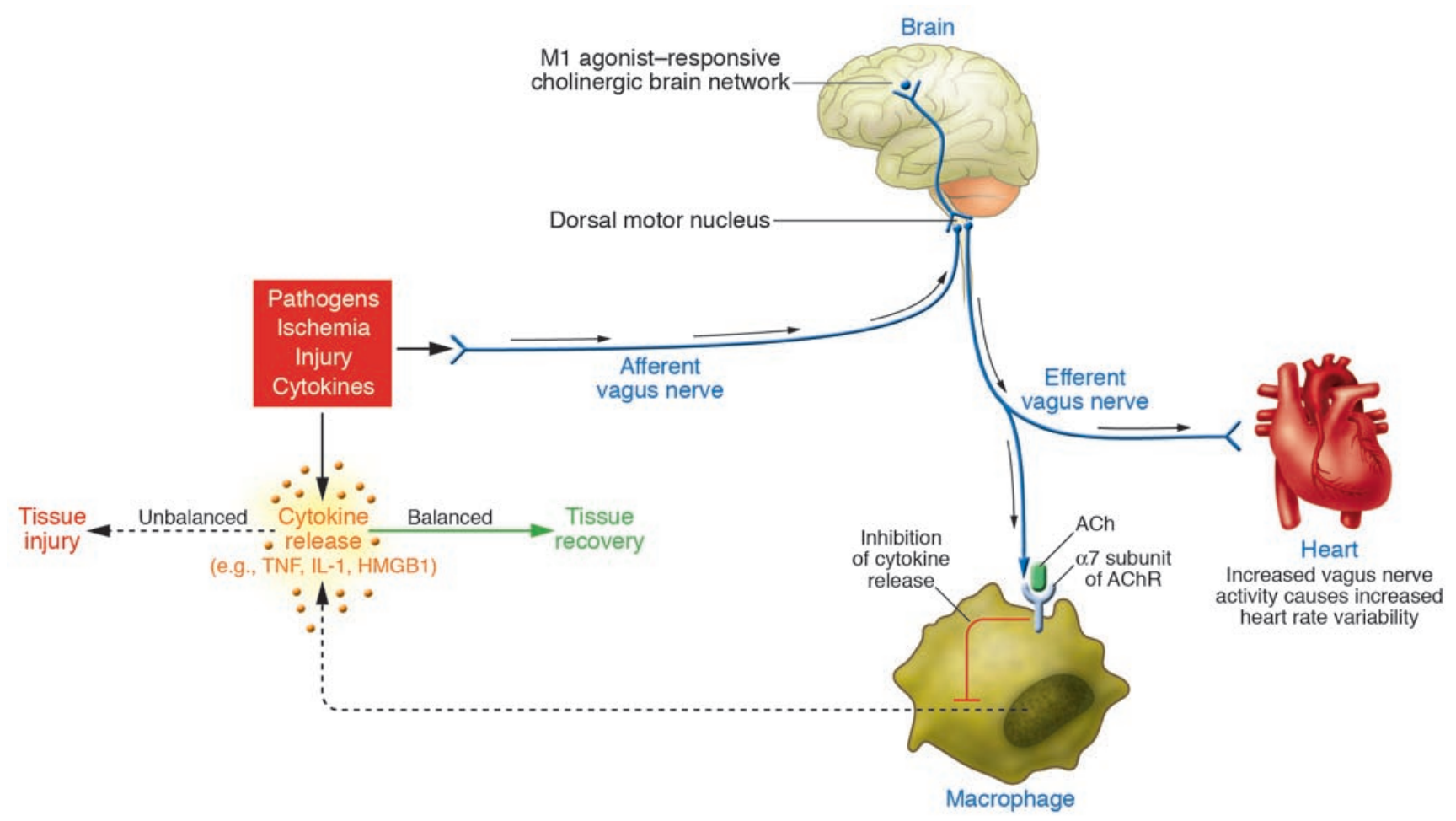

\section{Figure 2}

Wiring of the cholinergic antiinflammatory pathway, which balances cytokine production. Pathogens as well as ischemia and other forms of injury activate cytokine production, which normally restores health. If the cytokine response is unbalanced or excessive, however, then these same mediators can cause disease. Efferent signals from the vagus nerve, which can be controlled by brain networks, inhibit cytokine production via pathways dependent on the $\alpha 7$ subunit of the AChR on macrophages and other cells. Efferent vagus nerve activity also increases instantaneous heart rate variability. A cholinergic brain network that is responsive to M1 agonists can increase the activity of the cholinergic antiinflammatory pathway and also increase instantaneous heart rate variability. Afferent signals carried in the vagus nerve can activate an efferent response that inhibits cytokine release, termed the inflammatory reflex.

major vagus nerve neurotransmitter $(18,26,27)$. Macrophages and other cytokine-producing cells express acetylcholine receptors (AChRs), which transduce an intracellular signal that inhibits cytokine synthesis $(26,27)$. The best characterized of these cholinergic receptors that suppress cytokines is the $\alpha 7$ subunit of the nicotinic AChR ( $\alpha 7 \mathrm{nAChR})$. Mice exposed to endotoxin realize an unbalanced, excessive cytokine response characterized by exaggerated TNF levels if they are deficient in either the $\alpha 7 \mathrm{nAChR}$ subunit (as a result of genetic knockout) or vagus nerve activity (as a result of vagotomy) $(18,26)$. Moreover, vagus nerve stimulation in $\alpha 7 \mathrm{nAChR}-k n o c k o u t$ animals fails to suppress cytokine synthesis whereas it significantly inhibits cytokine release in wild-type littermates (26). This indicates that vagus nerve-derived cholinergic signals provide tonic or continuous neurological modulation of cytokine synthesis, functioning as a governor on an engine that limits the magnitude of the immune response.

Vagus nerve stimulation, or administration of $\alpha 7 \mathrm{nAChR}$ agonists, inhibits not only TNF but also IL-1, IL-6, IL-8, and highmobility group box 1 (HMGB1) (27). Signal transduction of $\alpha 7$ $\mathrm{nAChR}$ in neurons is modulated by ligand-gated ion channel functionality, but whether similar cellular mechanisms serve to control cytokine release is uncertain. Ligand-receptor interaction on cytokine-expressing cells culminates in decreased nuclear translocation of NF-KB as well as activation of the transcription factor STAT3 via phosphorylation by JAK2, which is recruited to the $\alpha 7$
nAChR (Figure 3) $(22,27)$. Ongoing studies should determine the answers to several important questions, including whether these downstream signaling mechanisms require cofactors, ion channel activity, or other specific substrates in immunologically competent cells. Nonetheless, together the present results indicate that $\alpha 7$ nAChR-dependent signals, which can be activated via vagus nerve stimulation, regulate cytokine production and the capacity for damage. The efferent arm of the inflammatory reflex, now termed the cholinergic antiinflammatory patbway (14), is a highly robust mechanism for cytokine control. Other immunologically competent cells (e.g., lymphocytes and microglia) express $\alpha 7 \mathrm{AChR}$, so it is an unexplored possibility that their function in autoimmune disease may be regulated by the cholinergic antiinflammatory pathway. This pathway can function in a precise manner analogous to the neurological regulation of heart rate and other visceral organ functions. As Lewis Thomas suggests, the cholinergic antiinflammatory pathway is a neurological mechanism that controls cytokines "with precision and restraint."

\section{It takes nerve to restrain cytokines: anatomy of an innervated cytokine system}

Recent studies of the physiology, functional anatomy, and cellular molecular mechanisms of the cholinergic antiinflammatory pathway indicate that the principal components for cytokine suppression by the vagus nerve converge in the spleen (28). Endotoxin 


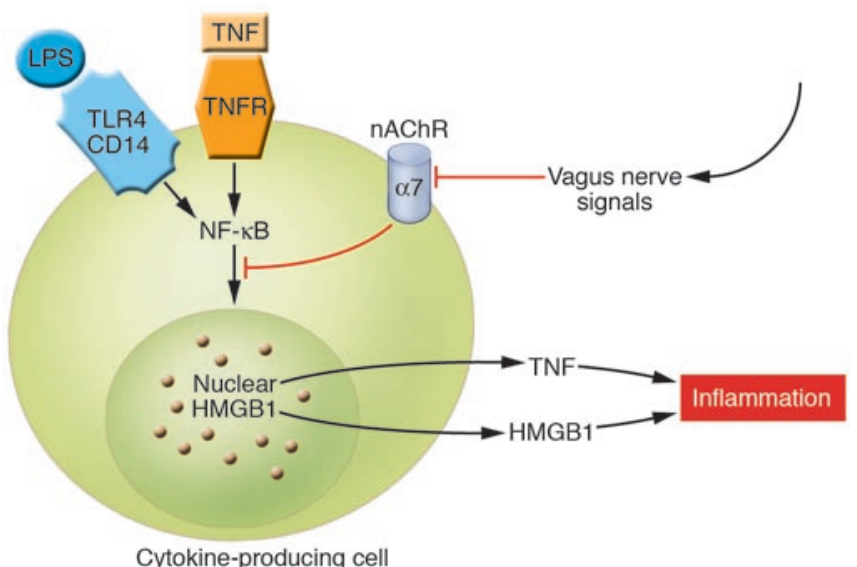

Figure 3

Cholinergic signaling mediated by $\alpha 7 \mathrm{nAChR}$ in cytokine-producing cells. Cholinergic signals derived from vagus nerve stimulation inhibit the release of TNF, IL-1, HMGB1, and other cytokines by transducing a cellular signal that inhibits the nuclear activity of NF-кB. TNFR, TNF receptor. Modified with permission from Nature Medicine (27).

localizes to macrophages primarily in the spleen and liver, thereby activating an immediate early cytokine response (29). The spleen is the major source of both hepatic and systemic TNF during endotoxemia; it releases newly synthesized TNF into the splenic vein, which drains into the liver, and from there, TNF crosses into the systemic circulation (28). Vagus nerve stimulation in WT mice inhibits TNF synthesis in spleen, liver, and blood but not in $\alpha 7$ nAChR subunit-knockout animals (28). Splenectomy significantly reduces TNF levels in liver and blood, and vagus nerve stimulation fails to reduce levels further (28). The functional anatomic mechanism of vagus nerve-derived signals to spleen traverses the common celiac branch of the vagus nerve because common celiac branch vagotomy abrogates the TNF-suppressing effects of vagus nerve stimulation in spleen and serum. These observations indicate the location of a critical interface for CNS control of TNF levels in spleen and also provide for the possibility that there might be a functional interface between the lymphoid compartments of spleen and the cholinergic antiinflammatory pathway.

Brain networks regulate the outflow of the cholinergic antiinflammatory pathway. Earlier work indicated that muscarinic brain networks can modulate vagus nerve regulation of other visceral functions. Examples include brain regulation of glycogen synthesis in the liver, of vasovagal syncope mediated by the Bezold-Jarisch cardiovascular reflex, and of exocrine pancreatic secretion (30-33). Recent results showing that intracerebroventricular administration of muscarine receptor agonists significantly decreases serum TNF levels during endotoxemia indicate that muscarinic brain networks also regulate the cytokine-controlling function of the vagus nerve. (34). Intracerebral administration of muscarine agonists also stimulates an increase in vagus nerve signaling to the heart as measured by increasing instantaneous heart rate variability, a result that couples the activation of a muscarinic brain network to two indicators of increased vagus nerve activity in the periphery: (a) increasing instantaneous heart rate variability and (b) decreasing cytokine production (34). It is interesting to consider that centrally activating muscarinic agents might be used as molecular tools to study and dissect the physiology and functional anatomy of the cholinergic antiinflammatory pathway along the path from the brain to the vagus nerve, across the common celiac branch, and into the spleen to prevent or modulate the development of cytokine-mediated disease.

\section{Preclinical efficacy of experimental therapeutics}

Preclinical studies are in progress to determine whether it may be possible to develop therapeutics based upon either devices that stimulate vagus nerve activity or drugs that activate the cholinergic antiinflammatory pathway to suppress cytokine damage. A significant number of studies indicate that the cholinergic antiinflammatory pathway is a robust regulator of cytokine-mediated damage in local and systemic experimental disease.

Sepsis. Cytokine imbalances mediate the development of organ damage and lethality during severe sepsis, a lethal syndrome that can develop after infection or injury. The cholinergic antiinflammatory pathway has been studied by several groups using animal models of sepsis in which a perforation is created in the cecum in order to induce lethal peritonitis. Treatment of these mice with nicotine, a potent $\alpha 7 \mathrm{nAChR}$ agonist, significantly reduced serum levels of HMGB1, a cytokine mediator of lethality in this model (27). Notably, nicotine administration did not begin until 24 hours after the onset of lethal peritonitis in this model, indicating that the cholinergic antiinflammatory pathway can control cytokine responses even when the disease process is already established (27). In a model of bacterial peritonitis based upon intraperitoneal injection of live Escherichia coli, vagotomy caused a significant increase in the number of inflammatory cells recruited to the peritoneum, higher cytokine levels, and more extensive tissue damage in the liver (35). Administration of nicotine to these animals restored the cytokine balance, reduced influx of inflammatory cells, and attenuated tissue damage in the liver (35). Other studies indicate that cholinergic stimulation controls endothelial activation, leukocyte recruitment, coagulation, and fibrinolysis, which modulates the development of damage during sepsis $(25,36)$.

Hemorrbagic shock. Cytokine overproduction has been implicated in the development of tissue damage and organ injury during hemorrhagic shock (37-39). Vagus nerve stimulation of rats during lethal hemorrhagic shock conferred significant protection against the development of hypotension, prolonged survival, and decreased TNF production (21). The protective effects of vagus nerve stimulation in this model were reversed by administration of a nicotine receptor antagonist (chlorisondamine), indicating that vagus nerve control of cytokine damage is attributable to cholinergic signals transduced intracellularly via nicotinic receptors (21). Another approach to stimulating efferent activity in the cholinergic antiinflammatory pathway in this model is by administration of adrenocorticotropic hormone $\left(\mathrm{ACTH}_{1-24}\right)$, which significantly increased vagus nerve activity in rats. Bilateral vagotomy ablated the protective effects of $\mathrm{ACTH}_{1-24}$ in hemorrhagic shock, providing evidence that $\mathrm{ACTH}_{1-24}$ activates proximal neural networks that increase efferent activity in the cholinergic antiinflammatory pathway to suppress cytokine-mediated damage (40). A high-fat diet also activates increased efferent vagus nerve activity, which decreases TNF levels and confers significant protection against hemorrhagic shock (41). The protective effects of dietary fat administration observed in this study were significantly reversed by vagotomy or by administering pharmacological agents that antagonized nicotinic receptors, providing evidence that fat consumption can specifically activate the cholinergic antiinflammatory pathway (41). 


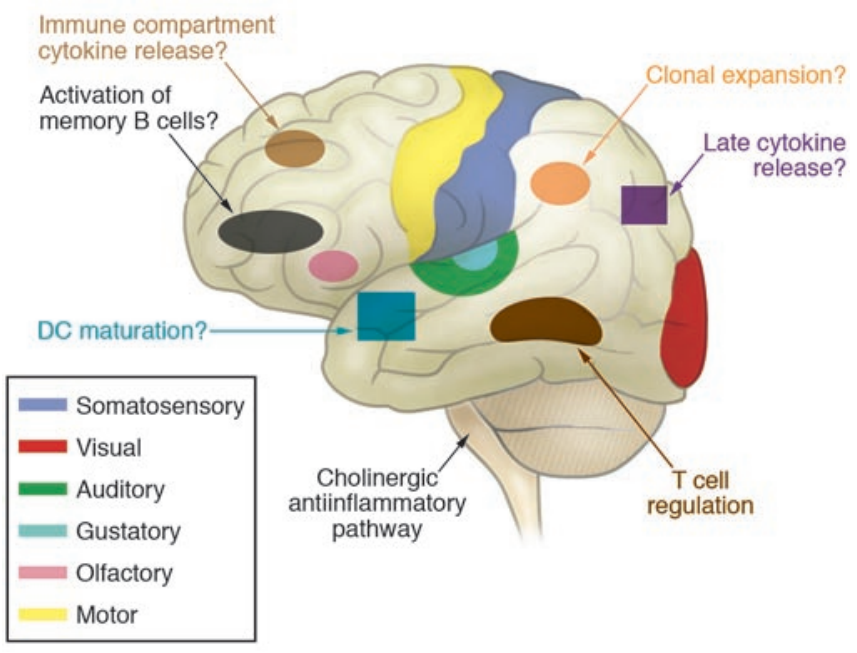

Figure 4

The immunological homunculus. The CNS is organized somatotopically, such that specific neural networks regulate or coordinate particular actions in the periphery. Advances in neuroimaging and neuroscience have made it possible to study this neural organization in humans. Knowledge about the cholinergic antiinflammatory pathway as a specific regulator of cytokine responses makes it possible to consider whether there is somatotopic organization to CNS regulation and coordination of the behavior of the immune system. It is plausible that this control extends beyond the simple regulation of cytokines. In the future, it may be possible to map specific brain regions that control other immune responses as depicted in this artistic rendition.

Ischemia/reperfusion. Reperfusion of ischemic tissues stimulates cytokine overproduction (e.g., TNF, IL-1, HMGB1), which contributes to the pathogenesis of resulting tissue damage (42-44). Vagus nerve stimulation significantly attenuated cytokine responses in a rat model of aortic ischemia/reperfusion injury (44). Intracerebral administration of CNI-1493, a tetravalent guanylhydrazone that stimulates muscarinic brain networks, increased vagus nerve activity and conferred significant protection against ischemia/ reperfusion-mediated damage $(17,44)$. The protective effects of intracerebral CNI-1493 required vagus nerve signals because vagotomy ablated the protective effects of administering the drug. In another model, vagus nerve stimulation conferred significant protection against the development of shock after splanchnic artery reperfusion injury and inhibited the production of TNF and other cytokines (20). Vagus nerve stimulation conferred protection against leukocyte accumulation in the ileum and lung and reduced the severity of tissue damage; the protective effects were reversed by administration of nicotinic receptor antagonists (20). Stimulation of the vagus nerve during myocardial ischemia/reperfusion in rats significantly reduced the accumulation of free radicals and reduced the incidence of severe arrhythmias and lethality (19). The protection against myocardial damage in this model was also achieved by administration of $\mathrm{ACTH}_{1-24}$, which stimulated a signal via the vagus nerve (19).

Pancreatitis. Previous work implicated cytokine overproduction in the pathogenesis of tissue damage and lethality in pancreatitis (45-47). Vagotomized mice with experimental pancreatitis developed significantly worsened tissue damage as compared with mice with intact vagus nerves. Administration of GTS-21, an $\alpha 7 \mathrm{nAChR}$ receptor agonist, significantly decreased pancreati- tis severity and attenuated pulmonary inflammation (24). This occurred even when the vagus nerves had been cut, indicating that a pharmacological approach to therapy may be useful even when the basal activity of the vagus nerve during the disease is insufficient to suppress cytokines.

Experimental arthritis. Vagus nerve stimulation has been studied in at least two models of localized inflammation in soft tissues. Stimulation of the vagus nerve in animals subjected to carrageenan-induced paw swelling significantly attenuated the development of edema formation and inhibited the local production of cytokines in the zone of inflammation (23). Vagus nerve stimulation of mice in a model of subcutaneous inflammation caused by injecting carrageenan into an air pouch significantly inhibited the recruitment of polymorphonuclear leukocytes to the inflammatory zone (25). As the vagus nerve does not signal directly to either the paw or the subcutaneous tissues, it is likely that the reduction in inflammation observed following vagus nerve stimulation in these models is attributable to the downregulation of cytokine production by the reticuloendothelial system and redirection of leukocyte trafficking away from the periphery (28).

Ileus. The pathogenesis of ileus, a complication of abdominal surgery or trauma characterized by gastrointestinal hypomotility and delayed gastric emptying, is mediated by the overproduction of cytokines in the bowel, which interferes with smooth muscle function $(48,49)$. Stimulation of the vagus nerve in mice with surgery-induced ileus significantly attenuated hypomotility and reduced cytokine production (22). Similar beneficial effects were achieved by administration of nicotine, which activated phosphorylation of STAT3 and its binding to and transactivation of its corresponding DNA-response elements. A role for $\alpha 7 \mathrm{nAChR}$ in the signal transduction cascade that suppressed cytokines was suggested by the observation that JAK2 was recruited to the $\alpha 7$ nAChR subunit (22).

\section{A wiring diagram as a template for the immunological homunculus}

The wiring diagram for the cholinergic antiinflammatory pathway (Figure 2) maps the concept that the brain can anatomically and functionally coordinate cytokine responses through neural networks that are analogous to other complex and essential physiological systems. It delineates a connection between brain networks and the immune system via a nerve that can be experimentally tracked, mapped, stimulated, or severed. It can be studied from beginning to end using classical techniques in neurophysiology, anatomy, and immunology. These can be combined with brain imaging studies, behavioral modification, and psychological interventions to test theories about the neurology of the immune response. I suspect that there exist structured, somatotopically organized neural networks that control specific components of the immune response by linking input to output. This theoretical organization is akin to the classic homunculus showing that specific brain regions exert control over specific body parts, and in the future it should be possible to construct an "immunological homunculus" (Figure 4). For example, one region of the brain may control cytokine responses in the liver and another control $\mathrm{T}$ cell activation in the spleen or lymph nodes. Certain centers might integrate knowledge about antigen presentation while others process input about dendritic cell maturation status. Distinct neurological domains within the CNS could regulate the state of overall readiness for innate responses to pathogens or trauma. The present 
neuroanatomical map of the cholinergic antiinflammatory pathway is a significant step toward defining other domains within the immunological homunculus that are crucial to maximizing host defense and maintaining health during immune responses.

The wiring diagram of the cholinergic antiinflammatory pathway provides a starting point from which we can begin to study hypotheses that are testable in animals and humans. From a practical point of view, the early evidence in this field suggests that it may be possible to develop experimental therapeutics based upon either (a) devices that directly stimulate the vagus nerve, (b) pharmacological agents that act on brain networks via muscarinic receptors to stimulate vagus nerve outflow, or (c) pharmacological agents that directly target the $\alpha 7$ receptor in the periphery to suppress cytokine production and reduce damage.

Abundant evidence points to the concept that complex behavior originating in higher brain centers can directly influence outflow through the vagus nerve. Sudden death, increased morbidity and mortality following cardiac surgery in hostile or depressed patients, and increased death rates in patients with sepsis or organ failure are linked clinically to decreased vagus nerve activity (50-54). Clinical research implicates a significant correlation between depressed vagus nerve activity and increased morbidity and mortality in sepsis, rheumatoid arthritis, lupus, sarcoidosis, inflammatory bowel diseases, other diseases, and trauma (55-63). These studies demonstrate that decreased vagus nerve activity is associated with increased disease severity, but they have not established a causal relationship. Depressed vagus nerve activity might facilitate the inflammatory responses underlying and worsening disease, but it remains possible that severe disease itself suppresses vagus nerve activity. The available evidence today may favor the former interpretation because deficiencies in the cholinergic antiinflammatory pathway lead to exaggerated cytokine responses and worsened morbidity. The final answer shall await clinical studies of vagus nerve modulation in humans to determine whether reversing vagus nerve deficiencies in these patients increases cholinergic antiinflammatory output and decreases cytokine levels and inflammatory disease, as it does in experimental animals.

\section{Shedding light on ancient concepts and modern clinical problems}

The physiology of the cholinergic antiinflammatory pathway can also be used to consider the design of clinical experiments for antiinflammatory therapies that were previously difficult to reconcile with classical mechanisms. Acupuncture, for instance, has been used for centuries for diseases as varied as inflammation and ischemia. The NIH fact sheet on acupuncture notes that "preclinical studies have documented acupuncture's effects, but they have not been able to fully explain how acupuncture works within the framework of the Western system of medicine" (64). It is difficult to reconcile the concepts of yin, yang, chi, and meridians with classical neurophysiological mechanisms. But mechanisms of the cholinergic antiinflammatory pathway enable developing hypotheses that are clinically testable using endpoints linked to vagus nerve activity, inflammatory responses, and disease severity. Acupuncture can elicit measurable increases in vagus nerve activity, so it is at least theoretically possible that this circuit can modulate the cytokine response via the cholinergic antiinflammatory pathway (65-68). Behavioral modification, meditation, hypnosis, biofeedback, and cognitive and relaxation therapies, which have been advocated for treatment of inflammation, can stimulate vagus nerve activity (69-75). It should now be possible to determine clinically whether these or other approaches activate the cholinergic antiinflammatory pathway.

On the pharmacology front, early clinical observations suggesting that cigarette smoking might be beneficial to patients with ulcerative colitis led to randomized clinical trials of nicotine administration to patients that demonstrated significant benefit in a subset of patients $(76,77)$. Although the protective mechanism was previously unknown, current understanding that nicotine inhibits cytokine production via an $\alpha 7 \mathrm{nAChR}$-dependent mechanism makes it plausible to consider whether this pathway is activated in the subset of patients that derive benefit from nicotine. Cigarette smoking confers some increased risk of the development of rheumatoid arthritis but is protective against osteoarthritis (78-80). Alzheimer disease and other brain degenerative disorders are characterized by cholinergic deficiency and decreased vagus nerve activity. Our recent evidence indicates that centrally acting cholinergic agonists used in the treatment of Alzheimer disease can modulate peripheral immune responses by stimulating brain networks to activate the cholinergic antiinflammatory pathway (34). It should be possible to determine whether these drugs modulate peripheral cytokine responses and vagus nerve activity in patients. Cholinergic and anticholinergic drugs are also widely used in intensive care units, where patients with sepsis and organ failure have increased cytokine levels and depressed vagus nerve activity $(55,56,81-83)$. It will be interesting to study whether commonly used cholinergic and anticholinergic drugs exert previously unrecognized effects on modulating cytokine responses, vagus nerve activity, and disease progression in this setting.

Truncal vagotomy was widely used in the past as a treatment for peptic ulcer disease, but whether this surgery altered cytokine responses is unknown. Perhaps this was simply not recognized, but it is also possible that with time the absence of vagus nerve control over cytokine responses activates other compensatory mechanisms. It will be important to learn whether compensation within the cholinergic antiinflammatory pathway occurs and whether activity in the pathway is subject to sensitization, desensitization, or tolerance, with important implications for developing therapeutic strategies. Similarly, it may be possible to study splenectomy patients to better understand the development of compensatory mechanisms to lesions in this pathway.

Exercise reduces levels of TNF and other cytokines, confers protection against cardiovascular disease and type 2 diabetes, increases vagus nerve activity, and confers protection against the development of atherosclerosis (84-90). It is possible that the mechanism of these exercise effects is at least in part attributable to exercise-induced increases in cholinergic antiinflammatory pathway activity. Obesity, on the other hand, is characterized by diminished vagus nerve output and elevated cytokine levels, which have been implicated in mediating insulin resistance and atherosclerosis (91-95). Since weight loss and exercise are each associated with increasing vagus nerve activity, one can consider whether enhanced activity in the cholinergic antiinflammatory pathway might decrease cytokine production and reduce the damage and metabolic derangements mediated by chronic, low-grade systemic inflammation that is characteristic of the metabolic syndrome.

Taking a step back from the reductionist approach and addressing the question of disease causation from a physiological viewpoint, it appears that we can learn lessons about balance in the milieu interior under vagus nerve control via the gene product of a 
specific AChR subunit. It is bemusing to think that one of the fundamental premises of the ancient Greeks was that dietary manipulation controlled humoral balances. This concept is now, at least in principle, supported by new evidence of a direct link between dietary composition and the regulation of cytokines by the cholinergic antiinflammatory pathway. Modern clinical studies have advocated supplementing diet with fish oil, soy oil, olive oil, and other fats to significantly increase vagus nerve activity, reduce inflammatory markers, and improve disease severity in inflammatory bowel disease, rheumatoid arthritis, and cardiovascular disease (91, 96-99). These clinical antiinflammatory responses may be linked to the fat-induced stimulation of the cholinergic antiinflammatory pathway, as is the case in rats (41). And now it appears that a major source of systemic TNF during lethal challenges is the spleen, the source of Galen's black bile (28). One can't help but wonder: How did the ancient Greeks know?

\section{Acknowledgments}

The author is grateful to Christopher Czura, Mitch Fink, Christine Metz, and Shaw Warren for helpful comments. Work in the author's laboratory is supported by the National Institute of General Medical Sciences at the NIH, the Defense Advanced Research Projects Agency, and Critical Therapeutics.

Address correspondence to: Kevin J. Tracey, Feinstein Institute for Medical Research, 350 Community Drive, Manhasset, New York 11030, USA. Phone: (516) 562-2813; Fax: (516) 562-2356; E-mail: kjtracey@nshs.edu.
1. Thomas, L. 1978. The lives of a cell: notes of a biology watcher. Penguin Books. New York, New York, USA 160 pp.

2. Tracey, K.J. 2005. Fatal sequence: the killer within. Dana Press. Washington, DC, USA. 238 pp.

3. Tracey, K.J., et al. 1987. Anti-cachectin/TNF monoclonal antibodies prevent septic shock during lethal bacteraemia. Nature. 330:662-664.

4. Tracey, K.J., et al. 1986. Shock and tissue injury induced by recombinant human cachectin. Science. 234:470-474.

5. Pfeffer, K., et al. 1993. Mice deficient for the 55 $\mathrm{kd}$ tumor necrosis factor receptor are resistant to endotoxic shock, yet succumb to L. monocytogenes infection. Cell. 73:457-467.

6. Dinarello, C.A., et al. 1986. Tumor necrosis factor (cachectin) is an endogenous pyrogen and induces production of interleukin 1. J. Exp. Med. 163:1433-1450.

7. Elliott, M.J., et al. 1994. Randomised double-blind comparison of chimeric monoclonal antibody to tumour necrosis factor alpha (cA2) versus placebo in rheumatoid arthritis. Lancet. 344:1105-1110.

8. Dinarello, C.A. 2004. Therapeutic strategies to reduce $\mathrm{IL}-1$ activity in treating local and systemic inflammation. Curr. Opin. Pharmacol. 4:378-385.

9. Targan, S.R., et al. 1997. A short-term study of chimeric monoclonal antibody $\mathrm{cA} 2$ to tumor necrosis factor alpha for Crohn's disease. Crohn's Disease cA2 Study Group. N. Engl. J. Med. 337:1029-1035.

10. Nathan, C. 2002. Points of control in inflammation. Nature. 420:846-852.

11. Davidson, N.J., et al. 1996. Thelper cell 1-type CD4+ $\mathrm{T}$ cells, but not B cells, mediate colitis in interleukin 10-deficient mice. J. Exp. Med. 184:241-251.

12. Johansson, A.C., Hansson, A.S., Nandakumar, K.S., Backlund, J., and Holmdahl, R. 2001. IL-10-deficient B10.Q mice develop more severe collageninduced arthritis, but are protected from arthritis induced with anti-type II collagen antibodies. J. Immunol. 167:3505-3512.

13. Sternberg, E.M., et al. 1989. Inflammatory mediator-induced hypothalamic-pituitary-adrenal axis activation is defective in streptococcal cell wall arthritis-susceptible Lewis rats. Proc. Natl. Acad. Sci. U. S. A. 86:2374-2378.

14. Tracey, K.J. 2002. The inflammatory reflex. Nature. 420:853-859.

15. Watkins, L.R., et al. 1995. Blockade of interleukin-1 induced hyperthermia by subdiaphragmatic vagotomy: evidence for vagal mediation of immunebrain communication. Neurosci. Lett. 183:27-31.

16. Hansen, M.K., O'Connor, K.A., Goehler, L.E., Watkins, L.R., and Maier, S.F. 2001. The contribution of the vagus nerve in interleukin-1beta-induced fever is dependent on dose. Am. J. Physiol. Regul. Integr. Comp. Physiol. 280:R929-R934.

17. Bernik, T.R., et al. 2002. Pharmacological stimulation of the cholinergic antiinflammatory pathway.
J. Exp. Med. 195:781-788.

18. Borovikova, L.V., et al. 2000. Vagus nerve stimulation attenuates the systemic inflammatory response to endotoxin. Nature. 405:458-462.

19. Mioni, C., et al. 2005. Activation of an efferent cholinergic pathway produces strong protection against myocardial ischemia/reperfusion injury in rats. Crit. Care Med. 33:2621-2628.

20. Altavilla, D., et al. 2006. Activation of the cholinergic antiinflammatory pathway reduces NF-kappab activation, blunts TNF-alpha production, and protects against splanchic artery occlusion shock. Shock. 25:500-506.

21. Guarini, S., et al. 2003. Efferent vagal fibre stimulation blunts nuclear factor-kappaB activation and protects against hypovolemic hemorrhagic shock. Circulation. 107:1189-1194.

22. de Jonge, W.J., et al. 2005. Stimulation of the vagus nerve attenuates macrophage activation by activating the Jak2-STAT3 signaling pathway. Nat. Immunol. 6:844-851.

23. Borovikova, L.V., et al. 2000. Role of vagus nerve signaling in CNI-1493-mediated suppression of acute inflammation. Auton. Neurosci. 85:141-147.

24. van Westerloo, D.J., et al. 2006. The vagus nerve and nicotinic receptors modulate experimental pancreatitis severity in mice. Gastroenterology. 130:1822-1830.

25. Saeed, R.W., et al. 2005. Cholinergic stimulation blocks endothelial cell activation and leukocyte recruitment during inflammation. J. Exp. Med. 201:1113-1123.

26. Wang, H., et al. 2003. Nicotinic acetylcholine receptor alpha7 subunit is an essential regulator of inflammation. Nature. 421:384-388.

27. Wang, H., et al. 2004. Cholinergic agonists inhibit HMGB1 release and improve survival in experimental sepsis. Nat. Med. 10:1216-1221.

28. Huston, J.M., et al. 2006. Splenectomy inactivates the cholinergic antiinflammatory pathway during lethal endotoxemia and polymicrobial sepsis. J. Exp. Med. 203:1623-1628.

29. Ge, Y., et al. 1997. Relationship of tissue and cellular interleukin-1 and lipopolysaccharide after endotoxemia and bacteremia. J. Infect. Dis. 176:1313-1321.

30. Shimazu, T., Matsushita, H., and Ishikawa, K. 1976. Cholinergic stimulation of the rat hypothalamus: effects of liver glycogen synthesis. Science. 194:535-536.

31. Saito, K., Yoshioka, M., Kohya, T., and Kitabatake, A 1994. Involvement of muscarinic M1 receptor in the central pathway of the serotonin-induced BezoldJarisch reflex in rats. J. Auton. Nerv. Syst. 49:61-68.

32. Li, Y., Wu, X., Zhu, J., Yan, J., and Owyang, C. 2003. Hypothalamic regulation of pancreatic secretion is mediated by central cholinergic pathways in the rat. J. Physiol. 552:571-587.

33. Wu, X., Gao, J., Yan, J., Owyang, C., and Li, Y. 2004. Hypothalamus-brain stem circuitry responsible for vagal efferent signaling to the pancreas evoked by hypoglycemia in rat. J. Neurophysiol. 91:1734-1747.

34. Pavlov, V.A., et al. 2006. Central muscarinic cholinergic regulation of the systemic inflammatory response during endotoxemia. Proc. Natl. Acad. Sci. U. S. A. 103:5219-5223.

35. van Westerloo, D.J., et al. 2005. The cholinergic antiinflammatory pathway regulates the host response during septic peritonitis. J. Infect. Dis. 191:2138-2148

36. van Westerloo, D.J., et al. 2006. Vagus nerve stimulation inhibits activation of coagulation and fibrinolysis during endotoxemia in rats. J. Thromb. Haemost. 4:1997-2002.

37. Tsung, A., et al. 2005. The nuclear factor HMGB1 mediates hepatic injury after murine liver ischemia-reperfusion. J. Exp. Med. 201:1135-1143.

38. Ertel, W., Morrison, M.H., Ayala, A., and Chaudry, I.H. 1994. Biological significance of elevated TNF levels: in vivo administration of monoclonal antibody against tnf following haemorrhage shock increases the capacity of macrophages to release TNF while restoring immunoresponsiveness. Cytokine. 6:624-632.

39. Zingarelli, B., et al. 1994. Role of tumor necrosis factor-alpha in acute hypovolemic hemorrhagic shock in rats. Am. J. Physiol. 266:H1512-H1515.

40. Guarini, S., et al. 2004. Adrenocorticotropin reverses hemorrhagic shock in anesthetized rats through the rapid activation of a vagal antiinflammatory pathway. Cardiovasc. Res. 63:357-365.

41. Luyer, M.D., et al. 2005. Nutritional stimulation of cholecystokinin receptors inhibits inflammation via the vagus nerve. J. Exp. Med. 202:1023-1029.

42. Izuishi, K., et al. 2006. Cutting edge: high-mobility group box 1 preconditioning protects against liver ischemia-reperfusion injury. J. Immunol. 176:7154-7158.

43. Colletti, L.M., et al. 1990. Role of tumor necrosis factor- $\alpha$ in the pathophysiologic alterations after hepatic ischemia/reperfusion injury in the rat. J. Clin. Invest. 85:1936-1943.

44. Bernik, T.R., et al. 2002. Cholinergic antiinflammatory pathway inhibition of tumor necrosis factor during ischemia reperfusion. J. Vasc. Surg. 36:1231-1236

45. Bhatia, M., et al. 2005. Pathophysiology of acute pancreatitis. Pancreatology. 5:132-144.

46. Denham, W., and Norman, J. 1999. The potential role of therapeutic cytokine manipulation in acute pancreatitis. Surg. Clin. North Am. 79:767-781.

47. Makhija, R., and Kingsnorth, A.N. 2002. Cytokine storm in acute pancreatitis. J. Hepatobiliary Pancreat. Surg. 9:401-410.

48. Bauer, A.J., Schwarz, N.T., Moore, B.A., Turler, A., and Kalff, J.C. 2002. Ileus in critical illness: mechanisms and management. Curr. Opin. Crit. Care. 8:152-157.

49. Bauer, A.J., and Boeckxstaens, G.E. 2004. Mecha- 
nisms of postoperative ileus. Neurogastroenterol. Motil. 16(Suppl. 2):54-60.

50. Lanza, G.A., et al. 1998. Prognostic role of heart rate variability in patients with a recent acute myocardial infarction. Am. J. Cardiol. 82:1323-1328.

51. Soejima, K., et al. 2000. Age-adjusted heart rate variability as an index of the severity and prognosis of heart failure. Jpn. Circ. J. 64:32-38.

52. Bilchick, K.C., et al. 2002. Prognostic value of heart rate variability in chronic congestive heart failure (Veterans Affairs' Survival Trial of Antiarrhythmic Therapy in Congestive Heart Failure). Am. J. Cardiol. 90:24-28.

53. Balanescu, S., Corlan, A.D., Dorobantu, M., and Gherasim, L. 2004. Prognostic value of heart rate variability after acute myocardial infarction. Med. Sci. Monit. 10:CR307-CR315.

54. Villareal, R.P., Liu, B.C., and Massumi, A. 2002. Heart rate variability and cardiovascular mortality. Curr. Atheroscler. Rep. 4:120-127.

55. Barnaby, D., et al. 2002. Heart rate variability in emergency department patients with sepsis. Acad. Emerg. Med. 9:661-670.

56. Pontet, J., et al. 2003. Heart rate variability as early marker of multiple organ dysfunction syndrome in septic patients. J. Crit. Care. 18:156-163.

57. Evrengul, H., et al. 2004. Heart rate variability in patients with rheumatoid arthritis. Rheumatol. Int 24:198-202.

58. Stein, K.S., McFarlane, I.C., Goldberg, N., and Ginzler, E.M. 1996. Heart rate variability in patients with systemic lupus erythematosus. Lupus. 5:44-48.

59. Laversuch, C.J., et al. 1997. Reduction in heart rate variability in patients with systemic lupus erythematosus. J. Rheumatol. 24:1540-1544.

60. Lindgren, S., Stewenius, J., Sjolund, K., Lilja, B., and Sundkvist, G. 1993. Autonomic vagal nerve dysfunction in patients with ulcerative colitis. Scand. J. Gastroenterol. 28:638-642.

61. Uslu, N., et al. 2006. Heart rate variability in patients with systemic sarcoidosis. Ann. Noninvasive Electrocardiol. 11:38-42.

62. Biswas, A.K., Scott, W.A., Sommerauer, J.F., and Luckett, P.M. 2000. Heart rate variability after acute traumatic brain injury in children. Crit. Care Med. 28:3907-3912.

63. Su, C.F., Kuo, T.B., Kuo, J.S., Lai, H.Y., and Chen, H.I. 2005. Sympathetic and parasympathetic activities evaluated by heart-rate variability in head injury of various severities. Clin. Neurophysiol. 116:1273-1279.

64. National Center for Complementary and Alternative Medicine, National Institutes of Health. 2004 NCCAM publication D003: Acupuncture. http:// nccam.nih.gov/health/acupuncture/.

65. Haker, E., Egekvist, H., and Bjerring, P. 2000. Effect of sensory stimulation (acupuncture) on sympathetic and parasympathetic activities in healthy subjects. J. Auton. Nerv. Syst. 79:52-59.

66. Shi, X., Wang, Z.P., and Liu, K.X. 1995. Effect of acupuncture on heart rate variability in coronary heart disease patients [In Chinese]. Zhongguo Zhong. Xi. Yi. Jie. He. Za Zhi. 15:536-538.

67. Chang, C.H., Huang, J.L., Ting, C.T., Chang, C.S., and Chen, G.H. 2005. Atropine-induced HRV alteration is not amended by electroacupuncture on Zusanli. Am. J. Chin. Med. 33:307-314.

68. Hsu, C.C., Weng, C.S., Liu, T.S., Tsai, Y.S., and Chang, Y.H. 2006. Effects of electrical acupuncture on acupoint BL15 evaluated in terms of heart rate variability, pulse rate variability and skin conductance response. Am. J. Chin. Med. 34:23-36.

69. Nolan, R.P., et al. 2005. Heart rate variability biofeedback as a behavioral neurocardiac intervention to enhance vagal heart rate control. Am. Heart J 149:1137.e1-1137.e7.

70. Palomba, D., Stegagno, L., and Zanchi, C. 1982. Biofeedback-assisted heart rate modification after myocardial infarction. J. Psychosom. Res. 26:469-473.

71. Cowan, M.J., Kogan, H., Burr, R., Hendershot, S., and Buchanan, L. 1990. Power spectral analysis of heart rate variability after biofeedback training. J. Electrocardiol. 23(Suppl.):85-94.

72. Sakakibara, M., Takeuchi, S., and Hayano, J. 1994 Effect of relaxation training on cardiac parasympathetic tone. Psychophysiology. 31:223-228.

73. Terathongkum, S., and Pickler, R.H. 2004. Relationships among heart rate variability, hypertension, and relaxation techniques. J. Vasc. Nurs. 22:78-82.

74. Peng, C.K., et al. 2004. Heart rate dynamics during three forms of meditation. Int. J. Cardiol. 95:19-27.

75. van Dixhoorn, J., and White, A. 2005. Relaxation therapy for rehabilitation and prevention in ischaemic heart disease: a systematic review and meta-analysis. Eur. J. Cardiovasc. Prev. Rehabil. 12:193-202.

76. Pullan, R.D., et al. 1994. Transdermal nicotine for active ulcerative colitis. N. Engl. J. Med. 330:811-815.

77. Sandborn, W.J., et al. 1997. Transdermal nicotine for mildly to moderately active ulcerative colitis. A randomized, double-blind, placebo-controlled trial. Ann. Intern. Med. 126:364-371.

78. Glossop, J.R., Dawes, P.T., and Mattey, D.L. 2006 Association between cigarette smoking and release of tumour necrosis factor $\alpha$ and its soluble receptors by peripheral blood mononuclear cells in patients with rheumatoid arthritis. Rheumatology (Oxford). 45:1223-1229.

79. Papadopoulos, N.G., et al. 2005. Does cigarette smoking influence disease expression, activity and severity in early rheumatoid arthritis patients? Clin. Exp. Rheumatol. 23:861-866.

80. Felson, D.T., et al. 1989. Does smoking protect against osteoarthritis? Arthritis Rheum. 32:166-172.

81. Schmidt, H.B., Werdan, K., and Muller-Werdan, U. 2001. Autonomic dysfunction in the ICU patient. Curr. Opin. Crit. Care. 7:314-322.

82. Schmidt, H., et al. 2005. Autonomic dysfunction predicts mortality in patients with multiple organ dysfunction syndrome of different age groups. Crit. Care Med. 33:1994-2002.

83. Korach, M., et al. 2001. Cardiac variability in critically ill adults: influence of sepsis. Crit. Care Med.
29:1380-1385

84. Jae, S.Y., et al. 2006. Effects of lifestyle modifications on C-reactive protein: contribution of weight loss and improved aerobic capacity. Metabolism. 55:825-831.

85. Goldhammer, E., et al. 2005. Exercise training modulates cytokines activity in coronary heart disease patients. Int. J. Cardiol. 100:93-99.

86. Obisesan, T.O., et al. 2004. C-reactive protein genotypes affect baseline, but not exercise training-induced changes, in C-reactive protein levels. Arterioscler. Thromb. Vasc. Biol. 24:1874-1879.

87. Barbeau, P., et al. 2002. Hemostatic and inflammatory markers in obese youths: effects of exercise and adiposity. J. Pediatr. 141:415-420.

88. Mattusch, F., Dufaux, B., Heine, O., Mertens, I., and Rost, R. 2000. Reduction of the plasma concentration of C-reactive protein following nine months of endurance training. Int. J. Sports Med. 21:21-24.

89. Lanza, G.A., et al. 2006. Relation of heart rate variability to serum levels of $\mathrm{C}$-reactive protein in patients with unstable angina pectoris. Am.J. Cardiol. 97:1702-1706.

90. Petersen, A.M., and Pedersen, B.K. 2005. The antiinflammatory effect of exercise. J. Appl. Physiol. 98:1154-1162.

91. Facchini, M., et al. 2003. Changes of autonomic cardiac profile after a 3-week integrated body weight reduction program in severely obese patients. J. Endocrinol. Invest. 26:138-142.

92. Akehi, Y., et al. 2001. VLCD-induced weight loss improves heart rate variability in moderately obese Japanese. Exp. Biol. Med. (Maywood). 226:440-445.

93. Minami, J., Kawano, Y., Ishimitsu, T., Matsuoka, H., and Takishita, S. 1999. Acute and chronic effects of a hypocaloric diet on 24-hour blood pressure, heart rate and heart-rate variability in mildly-to-moderately obese patients with essential hypertension. Clin. Exp. Hypertens. 21:1413-1427.

94. Gao, Y.Y., et al. 1996. Autonomic activity assessed by heart rate spectral analysis varies with fat distribution in obese women. Obes. Res. 4:55-63.

95. Ziegler, D., et al. 2006. Selective contribution of diabetes and other cardiovascular risk factors to cardiac autonomic dysfunction in the general population. Exp. Clin. Endocrinol. Diabetes. 114:153-159.

96. Holguin, F., et al. 2005. Cardiac autonomic changes associated with fish oil vs soy oil supplementation in the elderly. Chest. 127:1102-1107.

97. Berbert, A.A., Kondo, C.R., Almendra, C.L., Matsuo, T., and Dichi, I. 2005. Supplementation of fish oil and olive oil in patients with rheumatoid arthritis. Nutrition. 21:131-136.

98. Christensen, J.H., Christensen, M.S., Dyerberg, J., and Schmidt, E.B. 1999. Heart rate variability and fatty acid content of blood cell membranes: a doseresponse study with n-3 fatty acids. Am. J. Clin. Nutr. 70:331-337.

99. Christensen, J.H., Skou, H.A., Madsen, T., Torring, I., and Schmidt, E.B. 2001. Heart rate variability and n-3 polyunsaturated fatty acids in patients with diabetes mellitus. J. Intern. Med. 249:545-552. 